\title{
Cooperative Breast Cancer Tissue Resource
}

National Cancer Institute

\section{Source}

National Cancer Institute. Cooperative Breast Cancer Tissue Resource. NCI Thesaurus.

Code C16099.

Provides researchers with access to approximately 8,000 cases of formalin-fixed,

paraffin-embedded primary breast cancer specimens, with associated clinical data. The collection is particularly well-suited for validation studies of diagnostic and prognostic markers. 